\title{
Estabelecimento de normas DRIS para o algodoeiro com diferentes critérios de seleção da população de referência
}

\author{
Ademar Pereira Serra ${ }^{(1)}$, Marlene Estevão Marchetti(2), Enrique Pouyú Rojas( ${ }^{(3)}$, Henrique Soares de Morais ${ }^{(2)}$, \\ Vanessa do Amaral Conrad ${ }^{(2)}$ e Franklyn Clawdy Nunes Guimarães ${ }^{(2)}$
}

(1)Embrapa Gado de Corte, Caixa Postal 154, CEP 79002-970 Campo Grande, MS, Brasil. E-mail: ademar.serra@embrapa.br (2)Universidade Federal da Grande Dourados, Faculdade de Ciências Agrárias, Caixa Postal 533, CEP 79804-970 Dourados, MS, Brasil. E-mail: marlenemarchetti@ufgd.edu.br, ryck_1403@hotmail.com, nessa_conrad@hotmail.com, franklynclawdy@hotmail.com ${ }^{(3)}$ EPR Serviços Agrícolas, Avenida Cuiabá, no 829, Sala 83, Caixa Postal 22, CEP 78700-090 Rondonópolis, MT, Brasil. E-mail: epouyu@uol.com.br

Resumo - O objetivo deste trabalho foi avaliar o efeito de critérios de seleção de populações de referência no estabelecimento de normas DRIS na cultura do algodoeiro. Criou-se um banco de dados com a produtividade e os teores foliares de macro e micronutrientes, obtidos de talhões médios de 100 ha. Os critérios para o estabelecimento das subpopulações de alta produtividade foram talhões com: produtividade acima da média (4.380 $\left.\mathrm{kg} \mathrm{ha}^{-1}\right)$; produtividade acima da média $+2 / 3$ desvio-padrão (acima de $4.650 \mathrm{~kg} \mathrm{ha}^{-1}$ ); produtividade acima da média +1 desvio-padrão (acima de $4.785 \mathrm{~kg} \mathrm{ha}^{-1}$ ); e produtividade acima da média $+4 / 3$ desvio-padrão (acima de $4.920 \mathrm{~kg} \mathrm{ha}^{-1}$ ). Os critérios de seleção da população de referência proporcionam baixa frequência de relações nutricionais concordantes, para cada norma DRIS avaliada. Independentemente do critério de seleção utilizado, os dados de produtividade média e o índice de balanço nutricional relacionam-se significativamente; e essa relação intensifica-se com o aumento no rigor do critério de inclusão de lavouras na população de referência.

Termos para indexação: Gossypium hirsutum, alta produtividade, estado nutricional, índice de balanço nutricional, nutrição mineral, sistema integrado de diagnose.

\section{Establishing DRIS norms for cotton with different selection criteria for the reference population}

\begin{abstract}
The objective of this work was to evaluate the effect of selection criteria for reference populations on establishing DRIS norms for cotton crop. A database was created with data on yield and macro- and micronutrient contents in leaves obtained in average 100 ha plots. The criteria for establishing the high yield subpopulations were plots with: yield above average $\left(4,380 \mathrm{~kg} \mathrm{ha}^{-1}\right)$; yield above average $+2 / 3$ standard deviation (above 4,650 $\mathrm{kg} \mathrm{ha}^{-1}$ ); yield above average +1 standard deviation (above 4,785 $\mathrm{kg} \mathrm{ha}^{-1}$ ); and yield above average $+4 / 3$ standard deviation (above $4,920 \mathrm{~kg} \mathrm{ha}^{-1}$ ). The selection criteria for the reference population provide low agreement frequency between nutritional relations, for each evaluated DRIS norm. Independently of the criterion used, data on average yield and on the nutritional balance index are significantly related; and this relation is intensified with the increase in the rigor of criterion for the inclusion of crops in the reference population.
\end{abstract}

Index terms: Gossypium hirsutum, high productivity, nutritional status, nutritional balance index, mineral nutrition, diagnosis integrated system.

\section{Introdução}

O sistema integrado de diagnose e recomendação (DRIS), desenvolvido por Beaufils (1973), baseia-se no equilíbrio nutricional das plantas. Para diagnóstico desse balanço nutricional, o sistema gera índices para cada nutriente, em qualquer população de plantas que se deseje avaliar. Esses índices podem ser positivos ou negativos e, quanto mais próximos de zero, menor o desequilíbrio nutricional (excesso ou deficiência) do nutriente em avaliação.

À semelhança de outros métodos de avaliação do estado nutricional, o DRIS também requer a definição de valores de referência, os quais são denominados normas DRIS. A diferença para os métodos tradicionais reside na forma em que esses valores são definidos. Com o DRIS, eles podem ser obtidos com ampla variação nas condições ambientais (não há necessidade de 
controle), em lavouras comerciais e experimentais, ou podem ser específicos para determinada condição ou espaço geográfico (Silva et al., 2009; Serra et al., 2010a, 2010b, 2012).

Os índices DRIS são calculados a partir de relações bivariadas (normalmente quocientes) entre os teores foliares dos nutrientes. A função DRIS expressa a distância padronizada dessas relações para suas médias na população de referência. As normas DRIS consistem da média e do desvio-padrão das relações bivariadas entre os teores de nutrientes $(\mathrm{N} / \mathrm{P}, \mathrm{P} / \mathrm{K}$, $\mathrm{K} / \mathrm{Ca}$, etc.), na população de referência (Beaufils, 1973).

A definição das lavouras a serem utilizadas na definição da população de referência pode ter efeito sobre a eficácia das normas na diagnose nutricional (Escano et al., 1981; Walworth et al., 1988). A incerteza quanto a quais lavouras devem ser consideradas como referência agrava-se no momento de se decidir qual relação utilizar para compor as normas DRIS, se a direta $(\mathrm{A} / \mathrm{B})$ ou a inversa $(\mathrm{B} / \mathrm{A})$. Isso porque não há consenso quanto aos procedimentos para a escolha da forma a ser utilizada (direta ou inversa) no cálculo dos índices DRIS (Jones, 1981; Walworth \& Sumner, 1987; Ramakrishna et al., 2009).

A determinação da relação entre dois nutrientes quaisquer, portanto, pode ser feita em duas formas de expressão: direta e inversa (A/B ou B/A). Normalmente, tem-se utilizado a razão entre as variâncias das subpopulações de baixa e alta produtividade, para a escolha de qual forma da relação deve ser utilizada. Assim, recomenda-se a escolha da forma de expressão que proporcione maior razão de variâncias (Beaufils, 1973; Jones, 1981; Wadt et al., 1999; Serra et al., 2012). Esse procedimento favoreceria a escolha de relações com menor variação na subpopulação de alta produtividade.

O uso de diferentes critérios para a definição da população de referência pode resultar em normas distintas, com diferentes eficácias (Escano et al., 1981; Walworth et al., 1988; Guindani et al., 2009), com efeito direto na diagnose nutricional.

O objetivo deste trabalho foi avaliar o efeito de critérios de seleção de populações de referência no estabelecimento de normas DRIS na cultura do algodoeiro.

\section{Material e Métodos}

Foram utilizados dados de lavouras comerciais de algodão (Gossypium hirsutum), obtidos no ano agrícola 2004/2005, na região sul do Mato Grosso (12²41'S, $45^{\circ} 40^{\prime} \mathrm{W}$, com altitude média de $497 \mathrm{~m}$ ). O solo da região de estudo é predominantemente constituído por Latossolo Vermelho distroférrico (Santos et al., 2006). As lavouras foram conduzidas sob plantio convencional. As variedades que compuseram a base de dados foram: DeltaOpal, DeltaPenta, DeltaPine, Acala 90, CNPA ITA 90 e FiberMax 966.

Para compor a base de dados, foram utilizadas 108 amostras foliares compostas, cada uma formada por 30 amostras simples obtidas de plantas selecionadas aleatoriamente, em talhão comercial com área média de 100 ha. Em cada planta, foi coletada a folha (limbo + pecíolo) localizada na quinta posição, na haste principal (Malavolta, 2006), nos estádios fenológicos F1 a F4, segundo classificação de Marur \& Ruano (2001). As amostras foram secadas em estufa com circulação forçada de ar, a $65^{\circ} \mathrm{C}$, até peso constante, e passadas em moinho com peneira de 60 mesh. A produtividade de algodão em caroço $\left(\mathrm{kg} \mathrm{ha}^{-1}\right)$ foi avaliada com a colheita mecanizada dos talhões.

Nas amostras de folhas, foram determinados os teores totais de $\mathrm{N}, \mathrm{P}, \mathrm{K}, \mathrm{Ca}, \mathrm{Mg}, \mathrm{S}, \mathrm{B}, \mathrm{Zn}, \mathrm{Cu}, \mathrm{Mn}$ e Fe, conforme Malavolta et al. (1997). A base de dados para o desenvolvimento das normas DRIS foi formada pelos teores totais de macronutrientes $\left(\mathrm{g} \mathrm{kg}^{-1}\right)$ e micronutrientes $\left(\mathrm{mg} \mathrm{kg}^{-1}\right)$ nas folhas e pela produtividade de algodão em caroço $\left(\mathrm{kg} \mathrm{ha}^{-1}\right)$. A base foi dividida em quatro populações de referência, utilizando como critérios os níveis de produtividade das lavouras. As lavouras com produtividade inferior ao limite utilizado para a definição da população de referência foram consideradas como de baixa produtividade. A definição das populações de referência foi feita de acordo com quatro critérios: talhões com produtividade acima da média da população de lavouras amostradas; talhões com produtividade acima da média $+2 / 3$ desvio-padrão; talhões com produtividade acima da média +1 desvio-padrão; e talhões com produtividade acima da média $+4 / 3$ desvio-padrão.

Após a definição das populações de referência, a distribuição de normalidade das produtividades dos talhões selecionados foi testada com o teste de Kolmogorov-Smirnov (Campos, 1983). O teste foi

Pesq. agropec. bras., Brasília, v.48, n.11, p.1472-1480, nov. 2013 DOI: $10.1590 / \mathrm{S} 0100-204 X 2013001100008$ 
feito para cada subpopulação (talhão) e para o conjunto dos talhões amostrados.

Os teores dos nutrientes foram dispostos em relações bivariadas $(\mathrm{A} / \mathrm{B}$ ou $\mathrm{B} / \mathrm{A})$, tanto nas populações de referência quanto nas de baixa produtividade. A definição quanto à qual relação comporia as normas DRIS foi feita a partir da razão entre variâncias (valor $\mathrm{F}$ ) das relações bivariadas (A/B ou $\mathrm{B} / \mathrm{A}$ ), nas populações de baixa e alta produtividade, segundo Walworth \& Sumner (1987). Foi escolhida a forma da relação bivariada com maior razão de variâncias $\left(\mathrm{S}^{2}\right)$, de acordo com a seguinte análise: se $\mathrm{S}^{2}(\mathrm{~A} / \mathrm{B})_{\text {baixa produtividade }} / \mathrm{S}^{2}(\mathrm{~A} / \mathrm{B})_{\text {referência }}$ for maior que $\mathrm{S}^{2}(\mathrm{~B} / \mathrm{A})_{\text {baixa produtividade }} / \mathrm{S}^{2}(\mathrm{~B} / \mathrm{A})_{\text {referência, então a forma da }}$ relação A/B seria escolhida. Obviamente, se o inverso ocorresse, a relação $\mathrm{B} / \mathrm{A}$ seria escolhida.

Para testar as normas desenvolvidas de acordo com os quatro critérios de seleção da população de referência, os 108 talhões utilizados na base de dados foram agrupados em 11 classes de produtividade, com os respectivos teores médios de nutrientes nas folhas. O número de classes foi definido conforme Correa (2003) e Bataglia et al. (2004). Foram calculados os índices DRIS e os respectivos índices de balanço nutricional (IBN) para cada classe, e estudadas suas relações com a produtividade média dos talhões nessas classes.

As funções DRIS comparam as relações bivariadas entre dois nutrientes (ex: A/B) com as normas DRIS $(a / b)$, por meio de uma equação de estandardização. Utilizou-se a função de Beaufils (1973), com fator de ajuste $(\mathrm{K})=1$ : para $\mathrm{A} / \mathrm{B}<\mathrm{a} / \mathrm{b}$, $\mathrm{f}(\mathrm{A} / \mathrm{B})=100(1-\mathrm{a} / \mathrm{b} / \mathrm{B} / \mathrm{A}) \times \mathrm{K} / \mathrm{CV}(\%)$; e para $\mathrm{A} / \mathrm{B}>\mathrm{ab}, \mathrm{f}(\mathrm{A} / \mathrm{B})=100(\mathrm{~A} / \mathrm{B} / \mathrm{b} / \mathrm{a}-1) \times \mathrm{K} / \mathrm{CV}(\%)$.

Os índices DRIS foram calculados a partir da fórmula geral proposta por Beaufils (1973), em que, para o nutriente A: DRIS $\mathrm{A}=[\Sigma \mathrm{f}(\mathrm{A} / \mathrm{B})-\Sigma \mathrm{f}(\mathrm{B} / \mathrm{A})] /(\mathrm{n}+\mathrm{m})$, ou seja, o índice DRIS A resulta do somatório do valor das funções DRIS em que o nutriente aparece na relação direta, subtraído do somatório do valor das funções em que o nutriente aparece na relação inversa (B/A), dividido pela soma do número de funções em que o nutriente aparece na forma direta (n) ou inversa (m).

O IBN foi calculado por meio da soma do valor absoluto dos índices DRIS gerados na amostra (somatório em módulo dos índices), conforme Beaufils (1973).
Os IBN, gerados a partir das normas obtidas com os quatro critérios, passaram por um teste de identidade de modelos (Leite \& Oliveira, 2002) para avaliar a existência de semelhança entre as normas utilizadas. Neste processo, um critério foi considerado como padrão (Yi), e, então, comparado com os critérios alternativos $(\mathrm{Yj})$. Esse procedimento permite avaliar a identidade entre dois modelos analíticos por meio de uma combinação entre a estatística $\mathrm{F}\left[\mathrm{F}\left(\mathrm{H}_{0}\right)\right]$, na forma modificada por Graybill (1976), e o teste do erro médio $\left(t_{e}\right)$ do coeficiente de correlação linear entre os modelos $\left(\mathrm{r}_{\mathrm{YiYj}}\right)$.

Para tanto, ajustam-se os dados ao modelo $\mathrm{Y}_{\mathrm{j}}=\beta_{0}+\beta_{1} \mathrm{Y}_{\mathrm{i}}+\mathrm{e}$. A hipótese nula $\left(\mathrm{H}_{0}\right)$ pressupõe que $\beta_{0}=0$ e $\beta_{1}=1$. Essa hipótese foi testada a partir da estatística $\mathrm{F}\left(\mathrm{H}_{0}\right)$ de Graybill (1976). Para que houvesse identidade entre os modelos, a condição estabelecida era de aceitação da hipótese nula $\left(\mathrm{H}_{0}\right)$, não significância do teste $t_{e}$ e que $r_{Y i Y j}$ fosse maior que 1- | erro médio $\mid$.

Para verificar a homogeneidade de variância entre as subpopulações, utilizou-se o teste F unilateral, a $5 \%$ de probabilidade. As normas DRIS, obtidas a partir dos diferentes critérios de seleção da população de referência, foram comparadas duas a duas, pelo teste $\mathrm{t}$. As análises estatísticas foram conduzidas com o programa SPSS, para Windows, versão 11.0.0 (SPSS Inc., Chicago, IL, EUA). Os demais cálculos foram realizados com o uso da planilha do Excel (Microsoft Corporation, Redmond, WA, EUA).

\section{Resultados e Discussão}

Os valores médios das relações entre os teores dos nutrientes na população de referência, bem como seus respectivos coeficientes de variação, compõem as normas DRIS (Tabela 1). Com os 11 nutrientes avaliados, foi possível obter 55 relações selecionadas pelo método da razão entre variâncias. É comum a escolha das relações pelo método da razão entre as variâncias (Walworth \& Sumner, 1987; Wadt, 2005; Sema et al., 2010; Serra et al., 2012), uma vez que o método favorece a escolha de relações com menor variância na população de alta.

Os diferentes critérios de seleção da população de referência resultaram em 67,3 a 89,1\% de concordância na escolha da relação que comporia as normas (Tabela 2). No entanto, pôde-se constatar que, mesmo quando a relação escolhida para compor as normas era 
Tabela 1. Normas DRIS geradas pelos diferentes critérios de seleção da população de referência avaliados ${ }^{(1)}$.

\begin{tabular}{|c|c|c|c|c|c|c|c|c|c|c|c|}
\hline \multicolumn{3}{|c|}{ Critério 1} & \multicolumn{3}{|c|}{ Critério 2} & \multicolumn{3}{|c|}{ Critério 3} & \multicolumn{3}{|c|}{ Critério 4} \\
\hline Relação & Média & CV (\%) & Relação & Média & CV (\%) & Relação & Média & CV (\%) & Relação & Média & $\mathrm{CV}(\%)$ \\
\hline$\overline{\mathrm{N} / \mathrm{Ca}}$ & 1,6005 & 19,01 & $\mathrm{~N} / \mathrm{P}$ & 15,0928 & 12,89 & $\mathrm{~N} / \mathrm{Ca}$ & 1,4820 & 15,85 & $\mathrm{~N} / \mathrm{K}$ & 2,0859 & 11,79 \\
\hline $\mathrm{N} / \mathrm{S}$ & 4,8874 & 44,29 & $\mathrm{~N} / \mathrm{S}$ & 4,2504 & 50,11 & $\mathrm{~N} / \mathrm{S}$ & 3,8966 & 57,00 & $\mathrm{~N} / \mathrm{Ca}$ & 1,4661 & 18,68 \\
\hline N/B & 0,8039 & 32,73 & N/B & 0,7148 & 32,36 & N/B & 0,6485 & 31,79 & $\mathrm{~N} / \mathrm{S}$ & 4,0982 & 55,89 \\
\hline$N / Z n$ & 1,7334 & 20,89 & $\mathrm{~N} / \mathrm{Zn}$ & 1,6807 & 22,20 & $\mathrm{~N} / \mathrm{Zn}$ & 1,5559 & 19,60 & N/B & 0,6326 & 35,59 \\
\hline N/Mn & 1,0778 & 40,58 & N/Mn & 1,0520 & 38,03 & N/Mn & 1,1089 & 32,29 & $\mathrm{~N} / \mathrm{Zn}$ & 1,5388 & 25,31 \\
\hline $\mathrm{P} / \mathrm{N}$ & 0,0676 & 12,90 & $\mathrm{~N} / \mathrm{Fe}$ & 0,4869 & 30,80 & $\mathrm{~N} / \mathrm{Fe}$ & 0,4783 & 27,54 & N/Mn & 1,1236 & 42,91 \\
\hline $\mathrm{P} / \mathrm{S}$ & 0,3289 & 45,97 & $\mathrm{P} / \mathrm{S}$ & 0,2880 & 54,27 & $\mathrm{P} / \mathrm{N}$ & 0,0652 & 9,39 & $\mathrm{~N} / \mathrm{Fe}$ & 0,4795 & 26,14 \\
\hline $\mathrm{P} / \mathrm{B}$ & 0,0543 & 35,69 & $\mathrm{P} / \mathrm{B}$ & 0,0484 & 37,81 & $\mathrm{P} / \mathrm{K}$ & 0,1433 & 21,71 & $\mathrm{P} / \mathrm{N}$ & 0,0637 & 9,65 \\
\hline $\mathrm{P} / \mathrm{Zn}$ & 0,1164 & 21,65 & $\mathrm{P} / \mathrm{Mn}$ & 0,0691 & 33,06 & $\mathrm{P} / \mathrm{Ca}$ & 0,0966 & 18,55 & $\mathrm{P} / \mathrm{K}$ & 0,1330 & 15,94 \\
\hline $\mathrm{K} / \mathrm{N}$ & 0,4535 & 16,93 & $\mathrm{~K} / \mathrm{N}$ & 0,4580 & 16,32 & $\mathrm{P} / \mathrm{S}$ & 0,2552 & 60,82 & $\mathrm{P} / \mathrm{Ca}$ & 0,0923 & 11,94 \\
\hline $\mathrm{K} / \mathrm{P}$ & 6,8266 & 21,65 & $\mathrm{~K} / \mathrm{P}$ & 6,9248 & 21,50 & $\mathrm{P} / \mathrm{B}$ & 0,0427 & 36,70 & $\mathrm{P} / \mathrm{S}$ & 0,2545 & 52,29 \\
\hline $\mathrm{K} / \mathrm{S}$ & 2,1913 & 47,04 & $\mathrm{~K} / \mathrm{S}$ & 1,9068 & 48,53 & $\mathrm{P} / \mathrm{Zn}$ & 0,1006 & 16,46 & $\mathrm{P} / \mathrm{B}$ & 0,0406 & 40,32 \\
\hline $\mathrm{K} / \mathrm{B}$ & 0,3617 & 34,30 & $\mathrm{~K} / \mathrm{B}$ & 0,3251 & 35,06 & $\mathrm{P} / \mathrm{Mn}$ & 0,0714 & 29,33 & $\mathrm{P} / \mathrm{Zn}$ & 0,0968 & 20,75 \\
\hline $\mathrm{K} / \mathrm{Zn}$ & 0,7804 & 23,69 & $\mathrm{~K} / \mathrm{Zn}$ & 0,7610 & 23,00 & $\mathrm{P} / \mathrm{Fe}$ & 0,0315 & 31,89 & $\mathrm{P} / \mathrm{Cu}$ & 0,2554 & 60,21 \\
\hline $\mathrm{Ca} / \mathrm{P}$ & 9,6714 & 20,01 & $\mathrm{~K} / \mathrm{Mn}$ & 0,4806 & 41,15 & $\mathrm{~K} / \mathrm{N}$ & 0,4678 & 15,21 & $\mathrm{P} / \mathrm{Mn}$ & 0,0704 & 39,30 \\
\hline $\mathrm{Ca} / \mathrm{K}$ & 1,4485 & 20,01 & $\mathrm{Ca} / \mathrm{N}$ & 0,6600 & 17,62 & $\mathrm{~K} / \mathrm{S}$ & 1,8248 & 57,31 & $\mathrm{P} / \mathrm{Fe}$ & 0,0309 & 30,83 \\
\hline $\mathrm{Ca} / \mathrm{S}$ & 3,0128 & 39,73 & $\mathrm{Ca} / \mathrm{P}$ & 9,9640 & 21,65 & $\mathrm{~K} / \mathrm{B}$ & 0,3005 & 33,47 & $\mathrm{~K} / \mathrm{S}$ & 1,9781 & 57,04 \\
\hline $\mathrm{Ca} / \mathrm{Zn}$ & 1,0947 & 18,67 & $\mathrm{Ca} / \mathrm{K}$ & 1,4616 & 18,58 & $\mathrm{~K} / \mathrm{Mn}$ & 0,5252 & 38,74 & $\mathrm{~K} / \mathrm{B}$ & 0,3098 & 40,25 \\
\hline $\mathrm{Ca} / \mathrm{Fe}$ & 0,3218 & 26,60 & $\mathrm{Ca} / \mathrm{S}$ & 2,6471 & 41,79 & $\mathrm{Ca} / \mathrm{K}$ & 1,4983 & 17,00 & $\mathrm{~K} / \mathrm{Mn}$ & 0,5592 & 48,96 \\
\hline $\mathrm{Mg} / \mathrm{N}$ & 0,1004 & 22,21 & $\mathrm{Ca} / \mathrm{Zn}$ & 1,0855 & 19,69 & $\mathrm{Ca} / \mathrm{S}$ & 2,5646 & 49,21 & $\mathrm{Ca} / \mathrm{K}$ & 1,4574 & 17,41 \\
\hline $\mathrm{Mg} / \mathrm{P}$ & 1,4885 & 18,86 & $\mathrm{Ca} / \mathrm{Mn}$ & 0,6853 & 38,07 & $\mathrm{Ca} / \mathrm{B}$ & 0,4367 & 28,94 & $\mathrm{Ca} / \mathrm{S}$ & 2,7175 & 49,77 \\
\hline $\mathrm{Mg} / \mathrm{K}$ & 0,2282 & 28,63 & $\mathrm{Ca} / \mathrm{Fe}$ & 0,3166 & 29,07 & $\mathrm{Ca} / \mathrm{Zn}$ & 1,0629 & 20,61 & $\mathrm{Ca} / \mathrm{B}$ & 0,4379 & 38,06 \\
\hline $\mathrm{Mg} / \mathrm{Ca}$ & 0,1594 & 26,43 & $\mathrm{Mg} / \mathrm{N}$ & 0,0999 & 16,57 & $\mathrm{Ca} / \mathrm{Mn}$ & 0,7613 & 32,94 & $\mathrm{Ca} / \mathrm{Zn}$ & 1,0495 & 18,22 \\
\hline $\mathrm{Mg} / \mathrm{S}$ & 0,4850 & 51,41 & $\mathrm{Mg} / \mathrm{P}$ & 1,4944 & 15,29 & $\mathrm{Ca} / \mathrm{Fe}$ & 0,3319 & 30,00 & $\mathrm{Ca} / \mathrm{Mn}$ & 0,7751 & 41,74 \\
\hline $\mathrm{Mg} / \mathrm{B}$ & 0,0797 & 39,09 & $\mathrm{Mg} / \mathrm{K}$ & 0,2237 & 22,64 & $\mathrm{Mg} / \mathrm{N}$ & 0,1001 & 16,58 & $\mathrm{Ca} / \mathrm{Fe}$ & 0,3429 & 33,50 \\
\hline $\mathrm{Mg} / \mathrm{Zn}$ & 0,1717 & 26,50 & $\mathrm{Mg} / \mathrm{Ca}$ & 0,1555 & 22,67 & $\mathrm{Mg} / \mathrm{P}$ & 1,5394 & 14,89 & $\mathrm{Mg} / \mathrm{N}$ & 0,0991 & 12,81 \\
\hline $\mathrm{Mg} / \mathrm{Cu}$ & 0,4105 & 59,95 & $\mathrm{Mg} / \mathrm{S}$ & 0,4224 & 56,41 & $\mathrm{Mg} / \mathrm{K}$ & 0,2204 & 26,42 & $\mathrm{Mg} / \mathrm{P}$ & 1,5624 & 12,35 \\
\hline $\mathrm{Mg} / \mathrm{Mn}$ & 0,1047 & 39,35 & $\mathrm{Mg} / \mathrm{B}$ & 0,0709 & 37,68 & $\mathrm{Mg} / \mathrm{Ca}$ & 0,1478 & 22,29 & $\mathrm{Mg} / \mathrm{K}$ & 0,2093 & 24,33 \\
\hline $\mathrm{Mg} / \mathrm{Fe}$ & 0,0510 & 34,87 & $\mathrm{Mg} / \mathrm{Zn}$ & 0,1662 & 23,48 & $\mathrm{Mg} / \mathrm{S}$ & 0,3959 & 67,21 & $\mathrm{Mg} / \mathrm{Ca}$ & 0,1444 & 19,04 \\
\hline $\mathrm{B} / \mathrm{Ca}$ & 2,1669 & 29,72 & $\mathrm{Mg} / \mathrm{Cu}$ & 0,4234 & 52,04 & $\mathrm{Mg} / \mathrm{B}$ & 0,0639 & 34,37 & $\mathrm{Mg} / \mathrm{S}$ & 0,4036 & 57,16 \\
\hline $\mathrm{B} / \mathrm{S}$ & 6,3820 & 46,80 & $\mathrm{Mg} / \mathrm{Mn}$ & 0,1014 & 31,79 & $\mathrm{Mg} / \mathrm{Zn}$ & 0,1540 & 20,44 & $\mathrm{Mg} / \mathrm{B}$ & 0,0619 & 35,53 \\
\hline $\mathrm{B} / \mathrm{ZN}$ & 2,3409 & 32,09 & $\mathrm{Mg} / \mathrm{Fe}$ & 0,0487 & 32,83 & $\mathrm{Mg} / \mathrm{Cu}$ & 0,4056 & 55,17 & $\mathrm{Mg} / \mathrm{Zn}$ & 0,1505 & 22,62 \\
\hline $\mathrm{B} / \mathrm{Fe}$ & 0,6933 & 38,04 & $\mathrm{~B} / \mathrm{Ca}$ & 2,3503 & 25,56 & $\mathrm{Mg} / \mathrm{Mn}$ & 0,1076 & 27,49 & $\mathrm{Mg} / \mathrm{Cu}$ & 0,4051 & 62,40 \\
\hline $\mathrm{Zn} / \mathrm{S}$ & 2,8506 & 46,29 & $\mathrm{~B} / \mathrm{S}$ & 6,2165 & 53,30 & $\mathrm{Mg} / \mathrm{Fe}$ & 0,0482 & 31,99 & $\mathrm{Mg} / \mathrm{Mn}$ & 0,1077 & 36,84 \\
\hline $\mathrm{Cu} / \mathrm{N}$ & 0,3530 & 62,74 & $\mathrm{~B} / \mathrm{Fe}$ & 0,7352 & 35,50 & $\mathrm{~S} / \mathrm{B}$ & 0,2052 & 48,04 & $\mathrm{Mg} / \mathrm{Fe}$ & 0,0477 & 26,58 \\
\hline $\mathrm{Cu} / \mathrm{P}$ & 5,3300 & 65,98 & $\mathrm{Zn} / \mathrm{P}$ & 9,3474 & 22,49 & $\mathrm{~S} / \mathrm{Zn}$ & 0,5069 & 44,13 & $\mathrm{~S} / \mathrm{B}$ & 0,1949 & 55,68 \\
\hline $\mathrm{Cu} / \mathrm{K}$ & 0,7774 & 59,47 & $\mathrm{Zn} / \mathrm{S}$ & 2,5591 & 52,93 & $\mathrm{Zn} / \mathrm{K}$ & 1,4564 & 23,91 & $\mathrm{~S} / \mathrm{Zn}$ & 0,4609 & 39,33 \\
\hline $\mathrm{Cu} / \mathrm{Ca}$ & 0,5536 & 61,39 & $\mathrm{Zn} / \mathrm{B}$ & 0,4328 & 31,09 & $\mathrm{Zn} / \mathrm{B}$ & 0,4213 & 30,35 & $\mathrm{~S} / \mathrm{Mn}$ & 0,3597 & 53,35 \\
\hline $\mathrm{Cu} / \mathrm{S}$ & 1,7396 & 74,95 & $\mathrm{Zn} / \mathrm{Mn}$ & 0,6409 & 36,77 & $\mathrm{Cu} / \mathrm{N}$ & 0,3550 & 68,68 & $\mathrm{~B} / \mathrm{Zn}$ & 2,5742 & 22,91 \\
\hline $\mathrm{Cu} / \mathrm{B}$ & 0,2957 & 78,78 & $\mathrm{Cu} / \mathrm{N}$ & 0,3277 & 64,48 & $\mathrm{Cu} / \mathrm{P}$ & 5,4200 & 69,95 & $\mathrm{Zn} / \mathrm{K}$ & 1,4351 & 27,27 \\
\hline $\mathrm{Cu} / \mathrm{Zn}$ & 0,6242 & 73,11 & $\mathrm{Cu} / \mathrm{P}$ & 4,8722 & 66,38 & $\mathrm{Cu} / \mathrm{K}$ & 0,7507 & 62,01 & $\mathrm{Cu} / \mathrm{N}$ & 0,4026 & 80,78 \\
\hline $\mathrm{Mn} / \mathrm{P}$ & 17,1492 & 57,47 & $\mathrm{Cu} / \mathrm{K}$ & 0,7079 & 57,25 & $\mathrm{Cu} / \mathrm{Ca}$ & 0,5181 & 65,32 & $\mathrm{Cu} / \mathrm{K}$ & 0,8341 & 76,86 \\
\hline $\mathrm{Mn} / \mathrm{K}$ & 2,6188 & 60,26 & $\mathrm{Cu} / \mathrm{Ca}$ & 0,4969 & 59,06 & $\mathrm{Cu} / \mathrm{S}$ & 1,4179 & 89,78 & $\mathrm{Cu} / \mathrm{Ca}$ & 0,5736 & 77,56 \\
\hline $\mathrm{Mn} / \mathrm{Ca}$ & 1,8267 & 58,55 & $\mathrm{Cu} / \mathrm{S}$ & 1,4117 & 80,38 & $\mathrm{Cu} / \mathrm{B}$ & 0,2531 & 90,58 & $\mathrm{Cu} / \mathrm{S}$ & 1,5755 & 100,10 \\
\hline $\mathrm{Mn} / \mathrm{S}$ & 5,7560 & 84,66 & $\mathrm{Cu} / \mathrm{B}$ & 0,2405 & 79,59 & $\mathrm{Cu} / \mathrm{Zn}$ & 0,5766 & 83,29 & $\mathrm{Cu} / \mathrm{B}$ & 0,3020 & 104,85 \\
\hline $\mathrm{Mn} / \mathrm{B}$ & 0,8711 & 49,70 & $\mathrm{Cu} / \mathrm{Zn}$ & 0,5547 & 72,92 & $\mathrm{Cu} / \mathrm{Mn}$ & 0,4306 & 99,27 & $\mathrm{Cu} / \mathrm{Zn}$ & 0,6704 & 98,83 \\
\hline $\mathrm{Mn} / \mathrm{Zn}$ & 1,9688 & 60,67 & $\mathrm{Cu} / \mathrm{Mn}$ & 0,3611 & 96,58 & $\mathrm{Cu} / \mathrm{Fe}$ & 0,1898 & 90,17 & $\mathrm{Cu} / \mathrm{Mn}$ & 0,5170 & 116,13 \\
\hline $\mathrm{Mn} / \mathrm{Cu}$ & 4,7234 & 83,38 & $\mathrm{Cu} / \mathrm{Fe}$ & 0,1665 & 84,88 & $\mathrm{Mn} / \mathrm{S}$ & 4,9031 & 131,01 & $\mathrm{Mn} / \mathrm{B}$ & 0,6817 & 57,88 \\
\hline $\mathrm{Mn} / \mathrm{Fe}$ & 0,5830 & 61,08 & $\mathrm{Mn} / \mathrm{S}$ & 5,2110 & 103,44 & $\mathrm{Mn} / \mathrm{B}$ & 0,6651 & 54,88 & $\mathrm{Mn} / \mathrm{Zn}$ & 1,6770 & 60,77 \\
\hline $\mathrm{Fe} / \mathrm{N}$ & 2,1798 & 36,57 & $\mathrm{Mn} / \mathrm{B}$ & 0,7917 & 53,12 & $\mathrm{Mn} / \mathrm{Zn}$ & 1,5712 & 45,08 & $\mathrm{Fe} / \mathrm{K}$ & 4,6572 & 30,02 \\
\hline $\mathrm{Fe} / \mathrm{P}$ & 32,6525 & 38,57 & $\mathrm{Mn} / \mathrm{Fe}$ & 0,5611 & 60,91 & $\mathrm{Mn} / \mathrm{Fe}$ & 0,5214 & 70,64 & $\mathrm{Fe} / \mathrm{S}$ & 9,8300 & 83,96 \\
\hline $\mathrm{Fe} / \mathrm{K}$ & 4,9715 & 44,22 & $\mathrm{Fe} / \mathrm{P}$ & 34,7554 & 41,56 & $\mathrm{Fe} / \mathrm{K}$ & 5,1453 & 49,24 & $\mathrm{Fe} / \mathrm{B}$ & 1,3946 & 44,48 \\
\hline $\mathrm{Fe} / \mathrm{S}$ & 10,3607 & 59,05 & $\mathrm{Fe} / \mathrm{K}$ & 5,2424 & 49,59 & $\mathrm{Fe} / \mathrm{S}$ & 8,6275 & 68,16 & $\mathrm{Fe} / \mathrm{Zn}$ & 3,5813 & 58,83 \\
\hline $\mathrm{Fe} / \mathrm{Zn}$ & 3,6989 & 36,94 & $\mathrm{Fe} / \mathrm{S}$ & 9,4980 & 64,34 & $\mathrm{Fe} / \mathrm{B}$ & 1,4851 & 54,46 & $\mathrm{Fe} / \mathrm{Cu}$ & 10,2452 & 83,33 \\
\hline $\mathrm{Fe} / \mathrm{Cu}$ & 8,7290 & 65,82 & $\mathrm{Fe} / \mathrm{Zn}$ & 3,8258 & 42,29 & $\mathrm{Fe} / \mathrm{Zn}$ & 3,6406 & 49,20 & $\mathrm{Fe} / \mathrm{Mn}$ & 2,6233 & 69,94 \\
\hline
\end{tabular}

${ }^{(1)}$ Critério 1 , seleção de talhões com produtividade superior à média da população $(\mathrm{n}=60)$; critério 2 , talhões com produtividade superior à média $+2 / 3$ do desvio-padrão $(\mathrm{s})(\mathrm{n}=33)$; critério 3 , talhões com produtividade superior à média $+\mathrm{s}(\mathrm{n}=18)$; e critério 4 , talhões com produtividade superior à média $+4 / 3$ de $\mathrm{s}(\mathrm{n}=8)$. 
Tabela 2. Concordância entre as relações bivariadas escolhidas para compor as normas DRIS, de acordo com os diferentes critérios de seleção da população de referência, e comparação de seus valores médios.

\begin{tabular}{|c|c|c|c|c|c|}
\hline Critério 1 vs. 2 & Critério 1 vs. 3 & Critério 1 vs. 4 & Critério 2 vs. 3 & Critério 2 vs. 4 & Critério 3 vs. 4 \\
\hline \multicolumn{6}{|c|}{ Relações bivariadas } \\
\hline $\mathrm{N} / \mathrm{Ca}^{\ddagger}$ & $\mathrm{N} / \mathrm{Ca}^{* *}$ & $\mathrm{~N} / \mathrm{Ca}^{* *}$ & $\mathrm{~N} / \mathrm{P}^{\neq}$ & $\mathrm{N} / \mathrm{P}^{\neq}$ & $\mathrm{N} / \mathrm{Ca}^{\mathrm{ns}}$ \\
\hline $\mathrm{N} / \mathrm{S} * *$ & $\mathrm{~N} / \mathrm{S} * *$ & $\mathrm{~N} / \mathrm{S} *$ & $\mathrm{~N} / \mathrm{S}^{\mathrm{ns}}$ & $\mathrm{N} / \mathrm{S}^{\mathrm{ns}}$ & $\mathrm{N} / \mathrm{S}^{\mathrm{ns}}$ \\
\hline $\mathrm{N} / \mathrm{B}^{* *}$ & $\mathrm{~N} / \mathrm{B}^{* *}$ & $\mathrm{~N} / \mathrm{B}^{* *}$ & $\mathrm{~N} / \mathrm{B}^{* *}$ & $\mathrm{~N} / \mathrm{B}^{*}$ & $\mathrm{~N} / \mathrm{B}^{\mathrm{ns}}$ \\
\hline $\mathrm{N} / \mathrm{Zn}^{* *}$ & $\mathrm{~N} / \mathrm{Zn} * *$ & $\mathrm{~N} / \mathrm{Zn}^{* *}$ & $\mathrm{~N} / \mathrm{Zn} * *$ & $\mathrm{~N} / \mathrm{Zn} *$ & $\mathrm{~N} / \mathrm{Zn}^{\mathrm{ns}}$ \\
\hline $\mathrm{N} / \mathrm{Mn}^{\mathrm{ns}}$ & $\mathrm{N} / \mathrm{Mn}^{\mathrm{ns}}$ & $\mathrm{N} / \mathrm{Mn}^{\mathrm{ns}}$ & $\mathrm{N} / \mathrm{Mn}^{\mathrm{ns}}$ & $\mathrm{N} / \mathrm{Mn}^{\mathrm{ns}}$ & $\mathrm{N} / \mathrm{Mn}^{\mathrm{ns}}$ \\
\hline $\mathrm{P} / \mathrm{N}^{\ddagger}$ & $\mathrm{P} / \mathrm{N}^{* *}$ & $\mathrm{P} / \mathrm{N}^{* *}$ & $\mathrm{~N} / \mathrm{Fe}^{\text {ns }}$ & $\mathrm{N} / \mathrm{Fe}^{\text {ns }}$ & $\mathrm{N} / \mathrm{Fe}^{\mathrm{ns}}$ \\
\hline $\mathrm{P} / \mathrm{S} * *$ & $\mathrm{P} / \mathrm{S} * *$ & $\mathrm{P} / \mathrm{S} * *$ & $\mathrm{P} / \mathrm{S}^{*}$ & $\mathrm{P} / \mathrm{S}^{\mathrm{ns}}$ & $\mathrm{P} / \mathrm{N}^{\mathrm{ns}}$ \\
\hline $\mathrm{P} / \mathrm{B}^{* *}$ & $\mathrm{P} / \mathrm{B}^{* *}$ & $\mathrm{P} / \mathrm{B}^{* *}$ & $\mathrm{P} / \mathrm{B}^{* *}$ & $\mathrm{P} / \mathrm{B}^{* *}$ & $\mathrm{P} / \mathrm{K}^{\mathrm{ns}}$ \\
\hline $\mathrm{P} / \mathrm{Zn}^{\neq}$ & $\mathrm{P} / \mathrm{Zn} * *$ & $\mathrm{P} / \mathrm{Zn} * *$ & $\mathrm{P} / \mathrm{Mn}^{\mathrm{ns}}$ & $\mathrm{P} / \mathrm{Mn}^{\mathrm{ns}}$ & $\mathrm{P} / \mathrm{Ca}^{\mathrm{ns}}$ \\
\hline $\mathrm{K} / \mathrm{N}^{\mathrm{ns}}$ & $\mathrm{K} / \mathrm{N}^{* *}$ & $\mathrm{~K} / \mathrm{N}^{\ddagger}$ & $\mathrm{K} / \mathrm{N}^{\mathrm{ns}}$ & $\mathrm{K} / \mathrm{N}^{\ddagger}$ & $\mathrm{P} / \mathrm{S}^{\mathrm{ns}}$ \\
\hline $\mathrm{K} / \mathrm{P}^{\mathrm{ns}}$ & $\mathrm{K} / \mathrm{P}^{\neq}$ & $\mathrm{K} / \mathrm{P}^{\neq}$ & $\mathrm{K} / \mathrm{P}^{\neq}$ & $\mathrm{K} / \mathrm{P}^{\neq}$ & $\mathrm{P} / \mathrm{B}^{\mathrm{ns}}$ \\
\hline $\mathrm{K} / \mathrm{S} * *$ & $\mathrm{~K} / \mathrm{S} * *$ & $\mathrm{~K} / \mathrm{S}^{\mathrm{ns}}$ & $\mathrm{K} / \mathrm{S}^{\mathrm{ns}}$ & $\mathrm{K} / \mathrm{S}^{\mathrm{ns}}$ & $\mathrm{P} / \mathrm{Zn}^{\mathrm{ns}}$ \\
\hline $\mathrm{K} / \mathrm{B}^{* *}$ & $\mathrm{~K} / \mathrm{B}^{* *}$ & $\mathrm{~K} / \mathrm{B}^{* *}$ & $\mathrm{~K} / \mathrm{B}^{* *}$ & $\mathrm{~K} / \mathrm{B}^{\mathrm{ns}}$ & $\mathrm{P} / \mathrm{Mn}^{\mathrm{ns}}$ \\
\hline $\mathrm{K} / \mathrm{Zn} *$ & $\mathrm{~K} / \mathrm{Zn}^{\neq}$ & $\mathrm{K} / \mathrm{Zn}^{\neq}$ & $\mathrm{K} / \mathrm{Zn}^{\neq}$ & $\mathrm{K} / \mathrm{Zn}^{\neq}$ & $\mathrm{P} / \mathrm{Fe}^{\mathrm{ns}}$ \\
\hline $\mathrm{Ca} / \mathrm{P}^{* *}$ & $\mathrm{Ca} / \mathrm{P}^{\neq}$ & $\mathrm{Ca} / \mathrm{P}^{\neq}$ & $\mathrm{K} / \mathrm{Mn}^{*}$ & $\mathrm{~K} / \mathrm{Mn}^{*}$ & $\mathrm{~K} / \mathrm{N}^{\ddagger}$ \\
\hline $\mathrm{Ca} / \mathrm{K}^{\mathrm{ns}}$ & $\mathrm{Ca} / \mathrm{K}^{*}$ & $\mathrm{Ca} / \mathrm{K}^{\mathrm{ns}}$ & $\mathrm{Ca} / \mathrm{N}^{\ddagger}$ & $\mathrm{Ca} / \mathrm{N}^{\ddagger}$ & $\mathrm{K} / \mathrm{S}^{\mathrm{ns}}$ \\
\hline $\mathrm{Ca} / \mathrm{S}^{* *}$ & $\mathrm{Ca} / \mathrm{S}^{* *}$ & $\mathrm{Ca} / \mathrm{S}^{\text {ns }}$ & $\mathrm{Ca} / \mathrm{P}^{\neq}$ & $\mathrm{Ca} / \mathrm{P}^{\neq}$ & $\mathrm{K} / \mathrm{B}^{\mathrm{ns}}$ \\
\hline $\mathrm{Ca} / \mathrm{Zn}^{\mathrm{ns}}$ & $\mathrm{Ca} / \mathrm{Zn}^{*}$ & $\mathrm{Ca} / \mathrm{Zn}^{\mathrm{ns}}$ & $\mathrm{Ca} / \mathrm{K}^{\mathrm{ns}}$ & $\mathrm{Ca} / \mathrm{K}^{\mathrm{ns}}$ & $\mathrm{K} / \mathrm{Mn}^{\mathrm{ns}}$ \\
\hline $\mathrm{Ca} / \mathrm{Fe}^{\mathrm{ns}}$ & $\mathrm{Ca} / \mathrm{Fe}^{\mathrm{ns}}$ & $\mathrm{Ca} / \mathrm{Fe}^{\mathrm{ns}}$ & $\mathrm{Ca} / \mathrm{S}^{\text {ns }}$ & $\mathrm{Ca} / \mathrm{S}^{\text {ns }}$ & $\mathrm{Ca} / \mathrm{K}^{\mathrm{ns}}$ \\
\hline $\mathrm{Mg} / \mathrm{N}^{\mathrm{ns}}$ & $\mathrm{Mg} / \mathrm{N}^{\mathrm{ns}}$ & $\mathrm{Mg} / \mathrm{N}^{\mathrm{ns}}$ & $\mathrm{Ca} / \mathrm{Zn}^{\mathrm{ns}}$ & $\mathrm{Ca} / \mathrm{Zn}^{\mathrm{ns}}$ & $\mathrm{Ca} / \mathrm{S}^{\mathrm{ns}}$ \\
\hline $\mathrm{Mg} / \mathrm{P}^{\mathrm{ns}}$ & $\mathrm{Mg} / \mathrm{P}^{* *}$ & $\mathrm{Mg} / \mathrm{P}^{\mathrm{ns}}$ & $\mathrm{Ca} / \mathrm{Mn}^{* *}$ & $\mathrm{Ca} / \mathrm{Mn} *$ & $\mathrm{Ca} / \mathrm{B}^{\mathrm{ns}}$ \\
\hline $\mathrm{Mg} / \mathrm{K}^{\mathrm{ns}}$ & $\mathrm{Mg} / \mathrm{K}^{\mathrm{ns}}$ & $\mathrm{Mg} / \mathrm{K}^{*}$ & $\mathrm{Ca} / \mathrm{Fe}^{\mathrm{ns}}$ & $\mathrm{Ca} / \mathrm{Fe}^{\mathrm{ns}}$ & $\mathrm{Ca} / \mathrm{Zn}^{\mathrm{ns}}$ \\
\hline $\mathrm{Mg} / \mathrm{Ca} *$ & $\mathrm{Mg} / \mathrm{Ca} * *$ & $\mathrm{Mg} / \mathrm{Ca} *$ & $\mathrm{Mg} / \mathrm{N}^{\mathrm{ns}}$ & $\mathrm{Mg} / \mathrm{N}^{\mathrm{ns}}$ & $\mathrm{Ca} / \mathrm{Mn}^{\mathrm{ns}}$ \\
\hline $\mathrm{Mg} / \mathrm{S}^{* *}$ & $\mathrm{Mg} / \mathrm{S} * *$ & $\mathrm{Mg} / \mathrm{S}^{*}$ & $\mathrm{Mg} / \mathrm{P}^{*}$ & $\mathrm{Mg} / \mathrm{P}^{\mathrm{ns}}$ & $\mathrm{Ca} / \mathrm{Fe}^{\mathrm{ns}}$ \\
\hline $\mathrm{Mg} / \mathrm{B}^{* *}$ & $\mathrm{Mg} / \mathrm{B}^{* *}$ & $\mathrm{Mg} / \mathrm{B}^{* *}$ & $\mathrm{Mg} / \mathrm{K}^{\mathrm{ns}}$ & $\mathrm{Mg} / \mathrm{K}^{\mathrm{ns}}$ & $\mathrm{Mg} / \mathrm{N}^{\mathrm{ns}}$ \\
\hline $\mathrm{Mg} / \mathrm{Zn} * *$ & $\mathrm{Mg} / \mathrm{Zn} * *$ & $\mathrm{Mg} / \mathrm{Zn} * *$ & $\mathrm{Mg} / \mathrm{Ca} *$ & $\mathrm{Mg} / \mathrm{Ca} *$ & $\mathrm{Mg} / \mathrm{P}^{\mathrm{ns}}$ \\
\hline $\mathrm{Mg} / \mathrm{Cu}^{\mathrm{ns}}$ & $\mathrm{Mg} / \mathrm{Cu}^{\mathrm{ns}}$ & $\mathrm{Mg} / \mathrm{Cu}^{\mathrm{ns}}$ & $\mathrm{Mg} / \mathrm{S}^{\mathrm{ns}}$ & $\mathrm{Mg} / \mathrm{S}^{\text {ns }}$ & $\mathrm{Mg} / \mathrm{K}^{\mathrm{ns}}$ \\
\hline $\mathrm{Mg} / \mathrm{Mn}^{\mathrm{ns}}$ & $\mathrm{Mg} / \mathrm{Mn}^{\mathrm{ns}}$ & $\mathrm{Mg} / \mathrm{Mn}^{\mathrm{ns}}$ & $\mathrm{Mg} / \mathrm{B} * *$ & $\mathrm{Mg} / \mathrm{B}^{*}$ & $\mathrm{Mg} / \mathrm{Ca}^{\mathrm{ns}}$ \\
\hline $\mathrm{Mg} / \mathrm{Fe}^{* *}$ & $\mathrm{Mg} / \mathrm{Fe}^{*}$ & $\mathrm{Mg} / \mathrm{Fe}^{\mathrm{ns}}$ & $\mathrm{Mg} / \mathrm{Zn} * *$ & $\mathrm{Mg} / \mathrm{Zn} *$ & $\mathrm{Mg} / \mathrm{S}^{\mathrm{ns}}$ \\
\hline $\mathrm{B} / \mathrm{Ca}$ & $\mathrm{B} / \mathrm{Ca}^{\neq}$ & $\mathrm{B} / \mathrm{Ca}^{\neq}$ & $\mathrm{Mg} / \mathrm{Cu}^{\mathrm{ns}}$ & $\mathrm{Mg} / \mathrm{Cu}^{\mathrm{ns}}$ & $\mathrm{Mg} / \mathrm{B}^{\mathrm{ns}}$ \\
\hline $\mathrm{B} / \mathrm{S}^{\mathrm{ns}}$ & $\mathrm{B} / \mathrm{S}^{\neq}$ & $\mathrm{B} / \mathrm{S}^{\neq}$ & $\mathrm{Mg} / \mathrm{Mn} *$ & $\mathrm{Mg} / \mathrm{Mn}^{\mathrm{ns}}$ & $\mathrm{Mg} / \mathrm{Zn}^{\mathrm{ns}}$ \\
\hline $\mathrm{B} / \mathrm{Zn}^{\neq}$ & $\mathrm{B} / \mathrm{Zn}^{\neq}$ & $\mathrm{B} / \mathrm{Zn}^{\text {ns }}$ & $\mathrm{Mg} / \mathrm{Fe}^{\mathrm{ns}}$ & $\mathrm{Mg} / \mathrm{Fe}^{\mathrm{ns}}$ & $\mathrm{Mg} / \mathrm{Cu}^{\mathrm{ns}}$ \\
\hline $\mathrm{B} / \mathrm{Fe}^{* *}$ & $\mathrm{~B} / \mathrm{Fe}^{\neq}$ & $\mathrm{B} / \mathrm{Fe}^{\neq}$ & $\mathrm{B} / \mathrm{Ca}^{\neq}$ & $\mathrm{B} / \mathrm{Ca}^{\neq}$ & $\mathrm{Mg} / \mathrm{Mn}^{\mathrm{ns}}$ \\
\hline $\mathrm{Zn} / \mathrm{S}^{* *}$ & $\mathrm{Zn} / \mathrm{S}^{\neq}$ & $\mathrm{Zn} / \mathrm{S}^{\neq}$ & $\mathrm{B} / \mathrm{S}^{\neq}$ & $\mathrm{B} / \mathrm{S}^{\neq}$ & $\mathrm{Mg} / \mathrm{Fe}^{\mathrm{ns}}$ \\
\hline $\mathrm{Cu} / \mathrm{N}^{* *}$ & $\mathrm{Cu} / \mathrm{N}^{\mathrm{ns}}$ & $\mathrm{Cu} / \mathrm{N}^{\mathrm{ns}}$ & $\mathrm{B} / \mathrm{Fe}^{\neq}$ & $\mathrm{B} / \mathrm{Fe}^{\neq}$ & $\mathrm{S} / \mathrm{B}^{\mathrm{ns}}$ \\
\hline $\mathrm{Cu} / \mathrm{P}^{* *}$ & $\mathrm{Cu} / \mathrm{P}^{\mathrm{ns}}$ & $\mathrm{Cu} / \mathrm{P}$ & $\mathrm{Zn} / \mathrm{P}^{\neq}$ & $\mathrm{Zn} / \mathrm{P}^{\neq}$ & $\mathrm{S} / \mathrm{Zn}^{\text {ns }}$ \\
\hline $\mathrm{Cu} / \mathrm{K}^{* *}$ & $\mathrm{Cu} / \mathrm{K}^{\mathrm{ns}}$ & $\mathrm{Cu} / \mathrm{K}^{\mathrm{ns}}$ & $\mathrm{Zn} / \mathrm{S}^{\neq}$ & $\mathrm{Zn} / \mathrm{S}^{\neq}$ & $\mathrm{Zn} / \mathrm{K}^{\mathrm{ns}}$ \\
\hline $\mathrm{Cu} / \mathrm{Ca}^{* *}$ & $\mathrm{Cu} / \mathrm{Ca}^{\text {ns }}$ & $\mathrm{Cu} / \mathrm{Ca}^{\text {ns }}$ & $\mathrm{Zn} / \mathrm{B}^{\text {ns }}$ & $\mathrm{Zn} / \mathrm{B}^{\neq}$ & $\mathrm{Zn} / \mathrm{B}^{\neq}$ \\
\hline $\mathrm{Cu} / \mathrm{S}^{* *}$ & $\mathrm{Cu} / \mathrm{S} * *$ & $\mathrm{Cu} / \mathrm{S}^{\mathrm{ns}}$ & $\mathrm{Zn} / \mathrm{Mn}^{\neq}$ & $\mathrm{Zn} / \mathrm{Mn}^{\neq}$ & $\mathrm{Cu} / \mathrm{N}^{\mathrm{ns}}$ \\
\hline $\mathrm{Cu} / \mathrm{B}^{* *}$ & $\mathrm{Cu} / \mathrm{B}^{*}$ & $\mathrm{Cu} / \mathrm{B}^{\text {ns }}$ & $\mathrm{Cu} / \mathrm{N}^{\mathrm{ns}}$ & $\mathrm{Cu} / \mathrm{N}^{*}$ & $\mathrm{Cu} / \mathrm{P}^{\neq}$ \\
\hline $\mathrm{Cu} / \mathrm{Zn}^{* *}$ & $\mathrm{Cu} / \mathrm{Zn}^{\mathrm{ns}}$ & $\mathrm{Cu} / \mathrm{Zn}^{\mathrm{ns}}$ & $\mathrm{Cu} / \mathrm{P}^{\text {ns }}$ & $\mathrm{Cu} / \mathrm{P}^{\neq}$ & $\mathrm{Cu} / \mathrm{K}^{\mathrm{ns}}$ \\
\hline $\mathrm{Mn} / \mathrm{P}^{\ddagger}$ & $\mathrm{Mn} / \mathrm{P}^{\neq}$ & $\mathrm{Mn} / \mathrm{P}^{\neq}$ & $\mathrm{Cu} / \mathrm{K}^{\mathrm{ns}}$ & $\mathrm{Cu} / \mathrm{K}^{\mathrm{ns}}$ & $\mathrm{Cu} / \mathrm{Ca}^{\text {ns }}$ \\
\hline $\mathrm{Mn} / \mathrm{K}^{\ddagger}$ & $\mathrm{Mn} / \mathrm{K}^{\ddagger}$ & $\mathrm{Mn} / \mathrm{K}^{\ddagger}$ & $\mathrm{Cu} / \mathrm{Ca}^{\mathrm{ns}}$ & $\mathrm{Cu} / \mathrm{Ca}^{\mathrm{ns}}$ & $\mathrm{Cu} / \mathrm{S}^{\mathrm{ns}}$ \\
\hline $\mathrm{Mn} / \mathrm{Ca}^{\neq}$ & $\mathrm{Mn} / \mathrm{Ca}^{\ddagger}$ & $\mathrm{Mn} / \mathrm{Ca}^{\ddagger}$ & $\mathrm{Cu} / \mathrm{S}^{\mathrm{ns}}$ & $\mathrm{Cu} / \mathrm{S}^{\mathrm{ns}}$ & $\mathrm{Cu} / \mathrm{B}^{\mathrm{ns}}$ \\
\hline $\mathrm{Mn} / \mathrm{S} * *$ & $\mathrm{Mn} / \mathrm{S}^{*}$ & $\mathrm{Mn} / \mathrm{S}^{\neq}$ & $\mathrm{Cu} / \mathrm{B}^{\mathrm{ns}}$ & $\mathrm{Cu} / \mathrm{B}^{\mathrm{ns}}$ & $\mathrm{Cu} / \mathrm{Zn}^{\mathrm{ns}}$ \\
\hline $\mathrm{Mn} / \mathrm{B}^{* *}$ & $\mathrm{Mn} / \mathrm{B}^{*}$ & $\mathrm{Mn} / \mathrm{B}^{* *}$ & $\mathrm{Cu} / \mathrm{Zn}^{\mathrm{ns}}$ & $\mathrm{Cu} / \mathrm{Zn}^{\mathrm{ns}}$ & $\mathrm{Cu} / \mathrm{Mn}^{\mathrm{ns}}$ \\
\hline $\mathrm{Mn} / \mathrm{Zn}^{\neq}$ & $\mathrm{Mn} / \mathrm{Zn} * *$ & $\mathrm{Mn} / \mathrm{Zn}^{\mathrm{ns}}$ & $\mathrm{Cu} / \mathrm{Mn}^{*}$ & $\mathrm{Cu} / \mathrm{Mn}^{*}$ & $\mathrm{Cu} / \mathrm{Fe}^{\neq}$ \\
\hline $\mathrm{Mn} / \mathrm{Cu}^{\neq}$ & $\mathrm{Mn} / \mathrm{Cu}^{\neq}$ & $\mathrm{Mn} / \mathrm{Cu}^{\neq}$ & $\mathrm{Cu} / \mathrm{Fe}^{\mathrm{ns}}$ & $\mathrm{Cu} / \mathrm{Fe}^{\mp}$ & $\mathrm{Mn} / \mathrm{S}^{\neq}$ \\
\hline $\mathrm{Mn} / \mathrm{Fe}^{* *}$ & $\mathrm{Mn} / \mathrm{Fe}^{*}$ & $\mathrm{Mn} / \mathrm{Fe}^{\ddagger}$ & $\mathrm{Mn} / \mathrm{S}^{\mathrm{ns}}$ & $\mathrm{Mn} / \mathrm{S}^{\neq}$ & $\mathrm{Mn} / \mathrm{B}^{\mathrm{ns}}$ \\
\hline $\mathrm{Fe} / \mathrm{N}$ & $\mathrm{Fe} / \mathrm{N}^{\ddagger}$ & $\mathrm{Fe} / \mathrm{N}^{\neq}$ & $\mathrm{Mn} / \mathrm{B}^{* *}$ & $\mathrm{Mn} / \mathrm{B}^{*}$ & $\mathrm{Mn} / \mathrm{Zn}^{\mathrm{ns}}$ \\
\hline $\mathrm{Fe} / \mathrm{P}^{* *}$ & $\mathrm{Fe} / \mathrm{P}^{\neq}$ & $\mathrm{Fe} / \mathrm{P}^{\neq}$ & $\mathrm{Mn} / \mathrm{Fe}^{\mathrm{ns}}$ & $\mathrm{Mn} / \mathrm{Fe}^{\ddagger}$ & $\mathrm{Mn} / \mathrm{Fe}^{\neq}$ \\
\hline $\mathrm{Fe} / \mathrm{K}^{* *}$ & $\mathrm{Fe} / \mathrm{K}^{\mathrm{ns}}$ & $\mathrm{Fe} / \mathrm{K}^{\mathrm{ns}}$ & $\mathrm{Fe} / \mathrm{P}^{\neq}$ & $\mathrm{Fe} / \mathrm{P}^{\neq}$ & $\mathrm{Fe} / \mathrm{K}^{\mathrm{ns}}$ \\
\hline $\mathrm{Fe} / \mathrm{S} * *$ & $\mathrm{Fe} / \mathrm{S} * *$ & $\mathrm{Fe} / \mathrm{S}^{\mathrm{ns}}$ & $\mathrm{Fe} / \mathrm{K}^{\mathrm{ns}}$ & $\mathrm{Fe} / \mathrm{K}^{\mathrm{ns}}$ & $\mathrm{Fe} / \mathrm{S}^{\mathrm{ns}}$ \\
\hline $\mathrm{Fe} / \mathrm{Zn}^{\mathrm{ns}}$ & $\mathrm{Fe} / \mathrm{Zn}^{\mathrm{ns}}$ & $\mathrm{Fe} / \mathrm{Zn}^{\mathrm{ns}}$ & $\mathrm{Fe} / \mathrm{S}^{\mathrm{ns}}$ & $\mathrm{Fe} / \mathrm{S}^{\mathrm{ns}}$ & $\mathrm{Fe} / \mathrm{B}^{\mathrm{ns}}$ \\
\hline $\mathrm{Fe} / \mathrm{Cu}^{\neq}$ & $\mathrm{Fe} / \mathrm{Cu}^{\neq}$ & $\mathrm{Fe} / \mathrm{Cu}^{\text {ns }}$ & $\mathrm{Fe} / \mathrm{Zn}^{\mathrm{ns}}$ & $\mathrm{Fe} / \mathrm{Zn}^{\mathrm{ns}}$ & $\mathrm{Fe} / \mathrm{Zn}^{\text {ns }}$ \\
\hline \multicolumn{6}{|c|}{ Frequência de relações concordantes (\%) } \\
\hline $80 \%$ & $72,7 \%$ & $69,1 \%$ & $80 \%$ & $67,3 \%$ & $89,1 \%$ \\
\hline \multicolumn{6}{|c|}{ uência de relações com médias iguais pelo test } \\
\hline $23,64 \%$ & $23,64 \%$ & $41,82 \%$ & $52,73 \%$ & $47,27 \%$ & $89,09 \%$ \\
\hline
\end{tabular}

${ }^{n}$ Não significativo. * e**Relações bivariadas com médias significativamente diferentes, entre os critérios em comparação, na avaliação pelo teste t, a 5 e $1 \%$ de probabilidade, respectivamente. ${ }^{*}$ Relações (diretas ou inversas) não concordantes entre os critérios testados. 
a mesma, houve diferença entre as médias das normas obtidas pelos diferentes critérios.

As menores concordâncias entre as relações selecionadas para compor as normas foram obtidas com os critérios 1 e $2(23,64 \%)$, e 1 e $3(67,3 \%)$ (Tabela 2). A máxima concordância se deu com os critérios 3 e 4 (89,1\%). Em geral, os critérios de seleção da população de referência proporcionaram baixa frequência de relações concordantes, o que está de acordo com Khiari et al. (2001), que observaram que o critério utilizado para partição entre as subpopulações de referência e de baixa produtividade influencia a relação entre nutrientes escolhida para compor as normas. Essa influência pode alterar a eficácia do sistema e resultar em diagnósticos diferentes quanto ao estado nutricional das plantas, conforme Walworth et al. (1988).

Os IBN gerados pelas diferentes normas foram testados, dois a dois, para verificar se os modelos apresentavam identidade (Leite \& Oliveira, 2002). Os valores de $\mathrm{F}\left(\mathrm{H}_{0}\right)$ dos testes de identidade estiveram entre 8,418 e 28,120 , todos significativos a $1 \%$ de probabilidade. Assim, a hipótese alternativa - de que os coeficientes $\beta_{0}$ e $\beta_{1}$ eram diferentes de 0 e 1 , respectivamente - foi aceita.

O teste do erro médio $\left(t_{\mathrm{e}}\right)$ não foi significativo apenas na avaliação da identidade entre os critérios 2 e 4, e 3 e 4 . Neste caso, deve-se concluir que as diferenças observadas entre as normas obtidas por esses critérios foram aleatórias.

O coeficiente de correlação linear entre os modelos foi menor que 1- | erro médio | somente na comparação dos critérios 1 e 4,2 e 4 , e 3 e 4 . Além da aceitação da hipótese nula, para que houvesse identidade entre os modelos, também era necessário que o teste do erro médio $\left(\mathrm{t}_{\mathrm{e}}\right)$ não fosse significativo e que $\mathrm{r}_{\mathrm{YiYj}}$ fosse maior que 1-|erro médio| (Leite \& Oliveira, 2002). Essa condição não foi observada em nenhuma comparação entre os modelos (Tabela 3). Dessa forma, constatouse que não houve identidade entre as normas obtidas com os diferentes critérios testados, o que revela que os critérios de seleção da população de referência têm influência significativa sobre os índices de balanço nutricional obtidos.

Observou-se correlação negativa entre IBN e produtividade, em todos os critérios analisados (Figura 1). O aumento no rigor de seleção da população referência resultou em uma relação mais forte entre IBN e produtividade média dos talhões, divididos previamente em 11 classes de produtividade.

O método de Beaufils (1973) não prevê relação significativa entre produtividade e IBN. O diagrama de dispersão entre os dados de produtividade (y) e o IBN (x) apenas mostra que altos valores de IBN levam, necessariamente, a uma baixa produtividade. O diagrama mostra, ainda, que altas produtividades somente são obtidas com baixos IBN, mas que a ocorrência de um baixo valor deste não garante um alto valor daquela.

Dessa forma, Beaufils (1973) demonstrou que deve ser esperada alta variabilidade na produtividade de lavouras com baixo IBN (lavouras com produtividade alta e baixa), e baixa variabilidade na produtividade de lavouras com alto IBN (apenas lavouras com baixa produtividade), semelhante ao observado por Wadt et al. (1999) e Silveira et al. (2005).

No entanto, no presente trabalho, o uso dos teores médios dos nutrientes, em diferentes classes de produtividade, possibilitou o estabelecimento

Tabela 3. Resultados do teste de identidade dos modelos analíticos (Leite \& Oliveira, 2002) utilizados na comparação dos valores do índice de balanço nutricional (IBN) obtidos com as normas DRIS derivadas a partir de diferentes critérios de seleção da população de referência ${ }^{(1)}$.

\begin{tabular}{|c|c|c|c|c|c|c|c|c|}
\hline \multirow[t]{2}{*}{ Comparação } & \multicolumn{3}{|c|}{ Parâmetros dos modelos ${ }^{(2)}$} & \multirow[t]{2}{*}{ Erro médio (e) } & \multicolumn{3}{|c|}{ Parâmetros para avaliação da identidade ${ }^{(3)}$} & \multirow[t]{2}{*}{ Conclusão } \\
\hline & $\beta_{0}$ & $\beta_{1}$ & $\mathrm{r}_{\mathrm{yiyj}}$ & & $\mathrm{F}\left(\mathrm{H}_{0}\right)$ & Teste $t_{\mathrm{e}}$ & $\mathrm{r}_{\mathrm{yiyj}}>1-|\mathrm{e}|$ & \\
\hline Critério 1 vs. 2 & 0,09286 & $0,39176^{* *}$ & 0,9168 & 0,1429 & $20,473 * *$ & $6,625 * *$ & Sim & $\mathrm{Y}_{\mathrm{j}} \neq \mathrm{Y}_{\mathrm{i}}$ \\
\hline Critério 1 vs. 3 & 0,02658 & $1,81376^{* *}$ & 0,8748 & 0,2195 & $28,120 * *$ & $7,722 * *$ & Sim & $\mathrm{Y}_{\mathrm{j}} \neq \mathrm{Y}_{\mathrm{i}}$ \\
\hline Critério 1 vs. 4 & $-0,18613$ & $3,69540 * *$ & 0,7797 & 0,2011 & $19,859 * *$ & $6,104 * *$ & Não & $\mathrm{Y}_{\mathrm{j}} \neq \mathrm{Y}_{\mathrm{i}}$ \\
\hline Critério 2 vs. 3 & $-0,07661$ & $1,63611 * *$ & 0,9297 & 0,0736 & $8,418 * *$ & $4,089 * *$ & Sim & $\mathrm{Y}_{\mathrm{j}} \neq \mathrm{Y}_{\mathrm{i}}$ \\
\hline Critério 2 vs. 4 & $-0,33880$ & $4,40020 * *$ & 0,7551 & 0,0695 & $19,063 * *$ & $2,434^{\mathrm{ns}}$ & Não & $\mathrm{Y}_{\mathrm{j}} \neq \mathrm{Y}_{\mathrm{i}}$ \\
\hline Critério 3 vs. 4 & $-0,23187$ & $2,56960 * *$ & 0,8712 & $-0,0084$ & $16,336^{* *}$ & $0,466^{\mathrm{ns}}$ & Não & $\mathrm{Y}_{\mathrm{j}} \neq \mathrm{Y}_{\mathrm{i}}$ \\
\hline
\end{tabular}

${ }^{(1)}$ Critério 1 , seleção de talhões com produtividade superior à média da população $(n=60)$; critério 2 , talhões com produtividade superior à média $+2 / 3$ do desvio-padrão $(\mathrm{s})(\mathrm{n}=33)$; critério 3 , talhões com produtividade superior à média $+\mathrm{s}(\mathrm{n}=18)$; e critério 4 , talhões com produtividade superior à média $+4 / 3$ de s $(n=8) .{ }^{(2)} \beta_{0}$ e $\beta_{1}$, intercepto e coeficiente angular dos modelos; $r_{Y i Y j}$, coeficiente de correlação linear entre os modelos. ${ }^{(3)} \mathrm{F}\left(\mathrm{H}_{0}\right)$, valor $\mathrm{F}$ para a hipótese nula

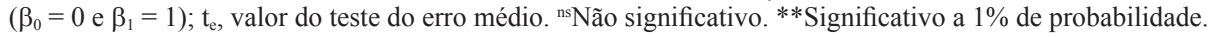



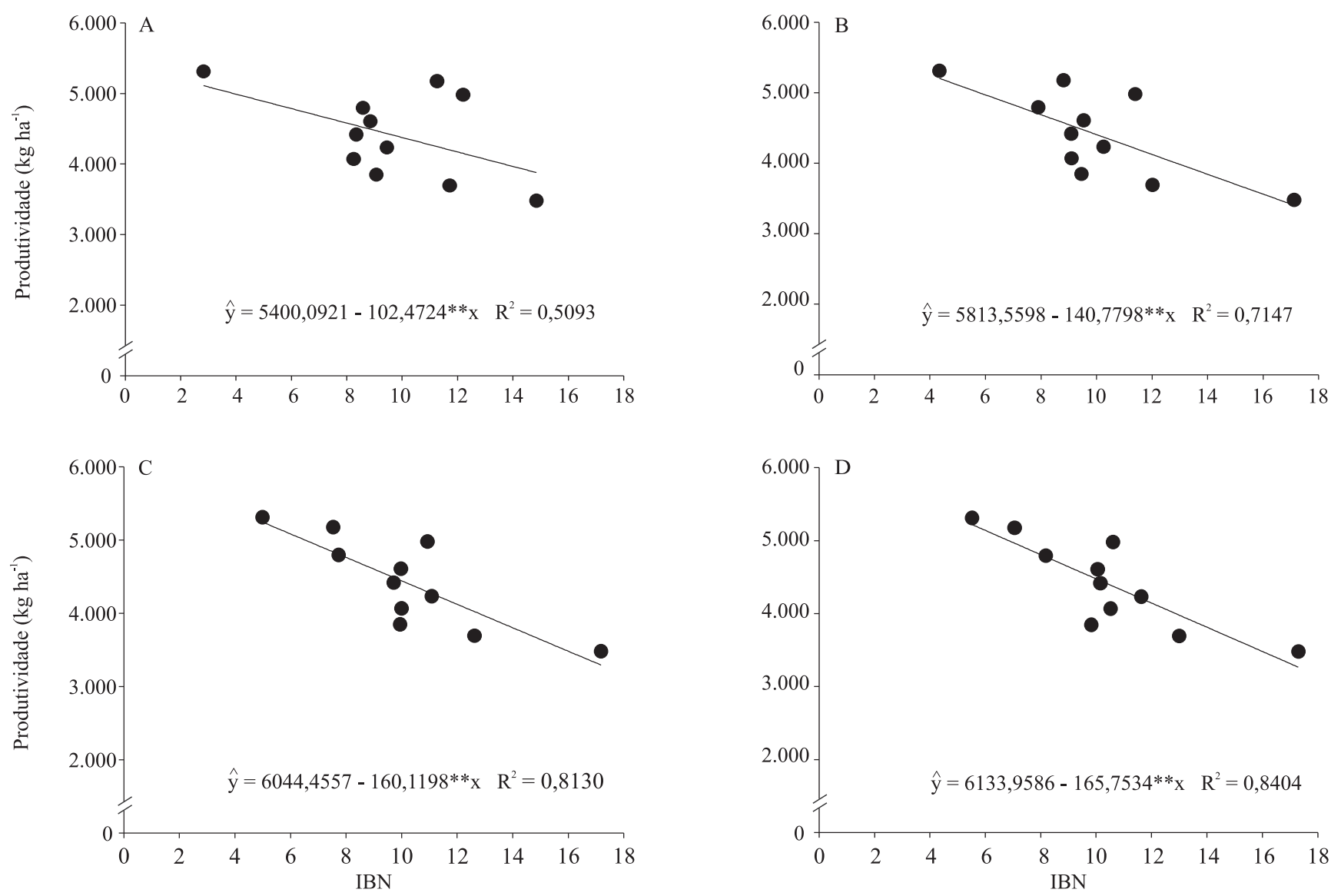

Figura 1. Relação entre os índices de balanço nutricional (IBN) e a produtividade, para os diferentes critérios de seleção da população de referência avaliados: critério 1 (A), seleção de talhões com produtividade superior à média da população $(n=60)$; critério $2(B)$, talhões com produtividade superior à média $+2 / 3$ do desvio-padrão $(s)(n=33)$; critério 3 (C), talhões com produtividade superior à média $+\mathrm{s}(\mathrm{n}=18)$; e critério $4(\mathrm{D})$, talhões com produtividade superior à média $+4 / 3$ de $\mathrm{s}(\mathrm{n}=8)$.

de relação mais forte entre IBN e produtividade. Essa distribuição em classes de produtividade foi previamente utilizada por Mourão Filho \& Azevedo (2003) e Silva et al. (2009), que também relataram alta relação entre IBN e produtividade. Neste caso, quanto mais forte a relação, mais associados estarão altos valores de IBN com baixa produtividade, e vice-versa.

De modo similar, correlações negativas entre IBN e produtividade foram observadas em trabalhos com número baixo de plantas no banco de dados, como em: Mourão Filho \& Azevedo (2003), com laranjeira 'Valência'; Bataglia et al. (2004), com cafeeiro; Nachtigall \& Dechen (2007), com macieira; Guindani et al. (2009), com arroz; e Silva et al. (2009), com algodão.

Os coeficientes de determinação $\left(\mathrm{R}^{2}\right)$ obtidos entre IBN e produtividade, no presente trabalho, podem ser considerados altos (Figura 1), uma vez que foram avaliadas lavouras comerciais, em que diferentes fatores influem na produção vegetal.

\section{Conclusões}

1. Os critérios de seleção da população de referência influenciam a relação bivariada entre teores de nutrientes a ser utilizada para compor as normas do sistema integrado de diagnose e recomendação (DRIS).

2. Não há identidade entre as normas geradas com os diferentes critérios testados e, portanto, os critérios de seleção têm influência significativa sobre a avaliação do estado nutricional das plantas.

3. Os dados médios de produtividade e índice de balanço nutricional (IBN) relacionam-se significativamente, independentemente do critério 
de seleção utilizado; e essa relação fica mais forte à medida que se aumenta o rigor do critério que define a inclusão de lavouras na população de referência.

\section{Agradecimentos}

À Coordenação de Aperfeiçoamento de Pessoal de Nível Superior (Capes), pela bolsa concedida.

\section{Referências}

BATAGLIA, O.C.; QUAGGIO, J.A.; SANTOS, W.R. dos; ABREU, M.F. de. Diagnose nutricional do cafeeiro pelo DRIS variando-se a constante de sensibilidade dos nutrientes de acordo com a intensidade e freqüência de resposta na produção. Bragantia, v.63, p.253-263, 2004. DOI: 10.1590/S0006-87052004000200010.

BEAUFILS, E.R. Diagnosis and recommendation integrated system (DRIS): a general scheme for experimentation and calibration based on principles developed from research in plant nutrition. Pietermararitzburg: University of Natal, 1973. 132p. (Soil science bulletin, 1).

CAMPOS, H. de. Estatística experimental não-paramétrica. 4.ed. Piracicaba: ESALQ, 1983. 349p.

CORREA, S.M.B.B. Probabilidade e estatística. 2.ed. Belo Horizonte: PUC Minas Virtual, 2003. 116p.

ESCANO, C.R.; JONES, C.A.; UEHARA, G. Nutrient diagnosis in corn grown on Hydric Dystrandepts. II. Comparison of two systems of tissue diagnosis. Soil Science Society of American Journal, v.45, p.1140-1144, 1981. DOI: 10.2136/sssaj1981.0361 5995004500060026x.

GRAYBILL, F.A. Theory and applications of the linear model. Belmonte: Wadsworth Publishing Company, 1976. 704p.

GUINDANI, R.H.P.; ANGHINONI, I.; NACHTIGALL, G.R. DRIS na avaliação do estado nutricional do arroz irrigado por inundação. Revista Brasileira de Ciência do Solo, v.33, p.109-118, 2009. DOI: 10.1590/S0100-06832009000100012.

JONES, C.A. Proposed modifications of the diagnosis and recommendation integrated system (DRIS) for interpreting plant analyses. Communications in Soil Science and Plant Analysis, v.12, p.785-794, 1981. DOI: 10.1080/00103628109367194.

KHIARI, L.; PARENT, L.T.; TREMBLAY, N. Selecting the high-yield subpopulation for diagnosing nutrient imbalance in crops. Agronomy Journal, v.93, p.802-808, 2001. DOI: 10.2134/ agronj2001.934802x.

LEITE, H.G.; OLIVEIRA, F.H.T. de. Statistical procedure to test identity between analytical methods. Communications in Soil Science and Plant Analysis, v.33, p.1105-1118, 2002. DOI: 10.1081/CSS-120003875.

MALAVOLTA, E. Manual de nutrição mineral de plantas. São Paulo: Agronômica Ceres, 2006. 638p.

MALAVOLTA, E.; VITTI, G.C.; OLIVEIRA, S.A. de. Princípios, métodos e técnicas de avaliação do estado nutricional. IN:
MALAVOLTA, E.; VITTI, G.C.; OLIVEIRA, S.A. de. Avaliação do estado nutricional de plantas: princípios e aplicações. 2.ed. Piracicaba: Potafos, 1997. p.115-230.

MARUR, C.J.; RUANO, O. A reference system for determination of developmental stages of upland cotton. Revista Brasileira de Oleaginosas e Fibrosas, v.5, p.313-317, 2001.

MOURÃO FILHO, F. de A.A.; AZEVEDO, J.C. DRIS norms for 'Valencia' sweet orange on three rootstocks. Pesquisa Agropecuária Brasileira, v.38, p.85-93, 2003. DOI: 10.1590/ S0100-204X2003000100012.

NACHTIGALL, G.R.; DECHEN, A.R. DRIS use on apple orchard nutritional evaluation in response to potassium fertilization. Communications in Soil Science and Plant Analysis, v.38, p.2557-2566, 2007. DOI: 10.1080/00103620701588882.

RAMAKRISHNA, A.; BAILEY, J.S.; KIRCHHOF, G. A preliminary diagnosis and recommendation integrated system (DRIS) model for diagnosing the nutrient status of sweet potato (Ipomoea batatas). Plant Soil, v.319, p.107-116, 2009. DOI: 10.1007/s11104-008-9763-5.

SANTOS, H.G. dos; JACOMINE, P.K.T.; ANJOS, L.H.C. dos; OLIVEIRA, V.A. de; OLIVEIRA, J.B. de; COELHO, M.R.; LUMBRERAS, J.F.; CUNHA, T.J.F. (Ed.). Sistema brasileiro de classificação de solos. 2.ed. Rio de Janeiro: Embrapa Solos, 2006. $306 \mathrm{p}$.

SEMA, A.; MAITI, C.S.; SINGH, A.K.; BENDANGSENGLA, A. DRIS nutrient norms for pineapple on alfisols of India. Journal of Plant Nutrition, v.33, p.1384-1399, 2010. DOI: 10.1080/01904167.2010.484286.

SERRA, A.P.; MARCHETTI, M.E.; ROJAS, E.P.; VITORINO, A.C.T. Beaufils ranges to assess the cotton nutrient status in the southern region of Mato Grosso. Revista Brasileira de Ciência do Solo, v.36, p.171-182, 2012. DOI: 10.1590/ S0100-06832012000100018.

SERRA, A.P.; MARCHETTI, M.E.; VITORINO, A.C.T.; NOVELINO, J.O.; CAMACHO, M.A. Desenvolvimento de normas DRIS e CND e avaliação do estado nutricional da cultura do algodoeiro. Revista Brasileira de Ciências do Solo, v.34, p.97-104, 2010a. DOI: 10.1590/S0100-06832010000100010.

SERRA, A.P.; MARCHETTI, M.E.; VITORINO, A.C.T.; NOVELINO, J.O.; CAMACHO, M.A. Determinação de faixas normais de nutrientes no algodoeiro pelos métodos CHM, CND e DRIS. Revista Brasileira de Ciência do Solo, v.34, p.105-113, 2010b. DOI: 10.1590/S0100-06832010000100011.

SILVA, M.A.C. da; NATALE, W.; MALHEIROS, E.B.; PAVINATO, A. Estabelecimento e validação de normas DRIS para a cultura do algodão no Centro-Oeste do Brasil. Acta Scientiarum. Agronomy, v.31, p.93-99, 2009. DOI: 10.4025/actasciagron. v31i1.6636.

SILVEIRA, C.P.; NACHTIGALL, G.R.; MONTEIRO, F.A. Testing and validation of methods for the diagnosis and recommendation integrated system for signal grass. Scientia Agricola, v.62, p.520-527, 2005. DOI: 10.1590/S0103-90162005000600002. 
WADT, P.G.S. Relationships between soil class and nutritional status of coffee plantations. Revista Brasileira de Ciência do Solo, v.29, p.227-234, 2005. DOI: 10.1590/S0100-06832005000200008.

WADT, P.G.S.; NOVAIS, R.F. de; ALVAREZ VENEGAS, V.H.; BRAGANÇA, S.M. Alternativas de aplicação do "DRIS" à cultura de café Conilon (Coffea canephora Pierre). Scientia Agricola, v.56, p.188-199, 1999. DOI: 10.1590/ S0103-90161999000100013.
WALWORTH, J.L.; SUMNER, M.E. The diagnosis and recommendation integrated system (DRIS). Advances in Soil Science, v.6,p.149-188, 1987.DOI: 10.1007/978-1-4612-4682-4_4.

WALWORTH, J.L.; WOODARD, H.J.; SUMNER, M.E. Generation of corn tissue norms from a small, high-yield data-base. Communications in Soil Science and Plant Analysis, v.19, p.563-577, 1988. DOI: $10.1080 / 00103628809367959$.

Recebido em 8 de agosto de 2013 e aprovado em 31 de outubro de 2013 